\title{
Preserving the baryon asymmetry after the electroweak phase transition with a $Z^{\prime}$
}

\author{
M.C. Bento ${ }^{\dagger}$ and O. Bertolami ${ }^{\dagger}$ \\ CERN, Theory Division \\ CH-1211 Geneva 23 \\ Switzerland
}

\begin{abstract}
Preservation of the baryon asymmetry after the electroweak phase transition is, within the Standard Model, barely compatible with the most recent lower bounds on the Higgs boson mass. We show that this situation can be considerably improved by considering extensions of the Standard Model with an extra neutral gauge boson state, $Z^{\prime}$, with no further onus to the physics of the electroweak phase transition.
\end{abstract}

CERN-TH.7049/93

October 1993

† On leave from Departamento de Física, Instituto Superior Técnico, Av. Rovisco Pais, 1096 Lisboa, Portugal 
1. Baryogenesis establishes a fundamental link between particle physics and cosmology as the conditions for the generation of any lasting and sizeable baryon asymmetry cannot be all found in either setting separately. Indeed, baryon and CP violating interactions can be found in various particle physics models but it is only in the early Universe that these interactions can occur out of thermal equilibrium [1].

Until fairly recently, baryon number violating interactions were thought to occur through the decay of heavy states either $\mathrm{i}$ at very high energies, in GUT models $[2,3]$, or at somewhat lower energies, in supersymmetric or superstring-inspired models [4], or through the thermalization of large vacuum energy of certain supersymmetric states $[5]^{(1)}$. Furthermore, the necessary CP violation was thought to be unrelated with the one existing in the Standard Model (SM).

The situation has taken a different turn after the realization that fermion number violation could occur at the electroweak phase transition, as speculated long ago [3], due to sphaleron-induced transitions between inequivalent non-abelian vacua [7], which are unsuppressed at finite temperature [8]. This alternative scenario requires the electroweak phase transition to be strongly first order and that bubbles with $\mathrm{SU}(3) \times \mathrm{U}(1)$ symmetry expand and percolate. Various mechanisms with these features have been outlined [9], the most promising one involving the so-called Charge Transport Mechanism, in which reflection and transmission of heavy fermions by the (CP violating) bubble walls gradually lead to a net baryon asymmetry [10]. The recent claim [11] that this mechanism can, in principle, generate the observed baryon asymmetry of the Universe (BAU) entirely within the SM (after a proper treatment of the various aspects of the fermion motion close to the bubble wall at finite temperature) is quite interesting and certainly deserves further study.

However, even if the baryon asymmetry can be generated at a stage as late as the electroweak phase transition, its survival depends crucially on the values of the Higgs and top quark masses. The requirement that B-violating transitions drop out of equilibrium

(1) For more exotic possibilities see ref. [6]. 
after the phase transition sets an upper bound on the Higgs mass, $m_{H}<64 \mathrm{GeV}$ [12], barely consistent with recent LEP searches [13], which establish that $m_{H}>58.4 \mathrm{GeV}^{(2)}$. In fact, recently discussed corrections to the SM effective potential at finite temperature, where higher loop corrections are included [14], the so-called ring diagrams, reveal that the electroweak phase transition is weaker first order than originally thought $[15,16]$, which thightens even further the upper bound on the Higgs boson mass [14]. It seems, therefore, that a way out of this difficulty necessarily involves some extension of the SM and, in fact, several models have already been analysed in the rerature [9]. Models with two Higgs doublets, although allowing for additional $\mathrm{CP}$ viol; $\mathrm{n}$, in general do not seem to be able to generate sufficient BAU [17]. The minimal SL JY extension of the standard model, while barely able to account for the BAU, has additional difficulties with baryon washout if there is any increase in the upper bound on the Higgs mass [18]. A recent proposal to avoid baryon washout involves the inclusion of an extra gauge singlet field [19] or actually several singlet fields [14].

In this letter, we assume that the necessary baryon asymmetry has been generated when the electroweak phase transition is completed, regardless of the scale at and the mechanism by which it has been produced, and we analyse the question of the preservation of the asymmetry after the electroweak phase transition in the simplest extension of the SM symmetry, namely the models with $S U(2) \times U(1) \times \tilde{U}(1)$ gauge symmetry at the $\mathrm{TeV}$ scale. Indeed, many extensions of the SM predict the existence of (at least) one additional gauge boson, $Z^{\prime}$, and some models allow $Z^{\prime}$ boson masses detectable at present or future colliders. Typical models are the ones based either on a left-right symmetry (LR) or $E_{6}$ (superstring-motivated or not) or $\mathrm{SO}(10)$, e.g. the so-called $\eta, \psi$ and $\chi$ models defined by the symmetry breaking schemes: $E_{6} \rightarrow S U(3) \times S U(2) \times U(1) \times U(1)_{\eta}$, $E_{6} \rightarrow S O(10) \times U(1)_{\psi}$ and $S O(10) \rightarrow S U(5) \times U(1)_{\chi} \times U(1)$, respectively (see $[20,21]$ and references therein). The extra gauge boson would manifest itself because the $\mathrm{Z}$ mass

(2) The combined bound of all LEP collaborations is $m_{H}>63 \mathrm{GeV}$. 
and couplings are modified by mixing and $Z^{\prime}$ exchange effects. The latter would also be responsible for the deformation of the $Z$ resonance shape; study of the shape of the resonance sets the limit $m_{Z^{\prime}} \gtrsim 100 \mathrm{GeV}[22]^{(3)}$. On the other hand, direct searches lead to $m_{Z^{\prime}} \gtrsim 400 \mathrm{GeV}$ [23], assuming standard model coupling strengths; for other models, e.g. the LR, $\eta, \psi$ and $\chi$ models mentioned above, bounds become less stringent varying typically between 170 and $350 \mathrm{GeV}$ [23]. In what follows, we shall not consider any model in particular but rather the class of models that admit a relatively "light" extra boson $Z^{\prime}$, i.e. with masses in the range $100-500 \mathrm{GeV}$, and assume that the remaining new scalar and fermionic states are heavy and decouple from the low energy physics.

We find that the contribution of the extra state, $Z^{\prime}$, to the effective potential enhances the first order features of the phase transition and allows upper limits on the Higgs boson mass which are compatible with experiment for $m_{Z^{\prime}} \gtrsim 200 \mathrm{GeV}$, for a broad range of top quark masses. Of course these bounds are further weakened as $m_{Z^{\prime}}$ increases and, for $m_{Z^{\prime}} \gtrsim 300 \mathrm{GeV}$, they become almost insensitive to the top quark mass. We also discuss the question of the validity of the thin wall approximation in these models and show that the amount of entropy released by the first order phase transition does not impose any further risk to the baryon asymmetry.

2. In models with an extra $U(1)$ symmetry, after symmetry breaking, the physical (mass eigenstate) vector bosons are

$$
\begin{aligned}
Z & =Z_{o} \cos \theta+Z_{o}^{\prime} \sin \theta \\
Z^{\prime} & =-Z_{o} \sin \theta+Z_{o}^{\prime} \cos \theta,
\end{aligned}
$$

where the lighter boson, $Z$, is the particle observed, $Z_{0}$ is the $\mathrm{SU}(2) \times \mathrm{U}(1)$ gauge boson which couples to the ordinary neutral current $g\left(J_{3 L}-\sin ^{2} \theta_{w} J_{e m}\right), Z_{o}^{\prime}$ couples to a new current $\tilde{g} \tilde{J}^{o}$ and $\theta$ is a mixing angle. Under these assumptions, and, for the mass range we

(3) In ref. [21], the mass bounds $m_{Z^{\prime}{ }_{x}}>900 \mathrm{GeV}$ and $500 \mathrm{GeV}<m_{Z^{\prime}{ }_{\eta}}<1 \mathrm{TeV}$ are obtained when allowing for an extra doublet of Higgs fields. 
are considering, i.e $100 \mathrm{GeV} \leq m_{Z^{\prime}} \leq 500 \mathrm{GeV}^{(4)}$, the finite temperature 1-loop effective potential, relevant to the discussion of the electroweak phase transition, is the following $[15,16,24]$ :

$$
V(\phi, T)=D\left(T^{2}-T_{o}^{2}\right) \phi^{2}-E T \phi^{3}+\frac{\lambda_{T}}{4} \phi^{4}
$$

where

$$
\begin{aligned}
& D= \frac{1}{8 \sigma^{2}}\left(2 m_{W}^{2}+m_{Z}^{2}+m_{Z^{\prime}}^{2}+2 m_{t}^{2}\right), \\
& E= \frac{1}{4 \pi \sigma^{3}}\left(2 m_{W}^{3}+m_{Z}^{3}+m_{Z^{\prime}}^{3}\right), \\
& T_{o}^{2}=\frac{1}{4 D}\left(m_{H}^{2}-8 B \sigma^{2}\right), \\
& B=\frac{3}{64 \pi^{2} \sigma^{4}}\left(2 m_{W}^{4}+m_{Z}^{4}+m_{Z^{\prime}}^{4}-4 m_{t}^{4}\right), \\
& \lambda_{T}=\lambda-\frac{3}{16 \pi^{2} \sigma^{4}}\left[2 m_{W}^{4} \ln \left(\frac{m_{W}^{2}}{a_{B} T^{2}}\right)+m_{Z}^{4} \ln \left(\frac{m_{Z}^{2}}{a_{B} T^{2}}\right)\right. \\
&\left.\quad+m_{Z^{\prime}}^{4} \ln \left(\frac{m_{Z^{\prime}}^{2}}{a_{B} T^{2}}\right)-4 m_{t}^{4} \ln \left(\frac{m_{t}^{2}}{a_{F} T^{2}}\right)\right]
\end{aligned}
$$

with $m_{H}^{2}=2 \lambda \sigma^{2}, \sigma=246 \mathrm{GeV}, \ln a_{B} \simeq 3.91$ and $\ln a_{F} \simeq 1.14$.

To $V(\phi, T)$, one has to add the ring diagram contributions $[15,16]$ :

$$
\begin{aligned}
V_{R D}(\phi, T)=-\frac{T}{12 \pi} & \left(2\left[m_{W}^{2}(\phi)+\Pi_{W}^{o o}(0)\right]^{3 / 2}+\left[m_{Z}^{2}(\phi)+\Pi_{Z}^{o o}(0)\right]^{3 / 2}\right. \\
& \left.+\left[m_{Z^{\prime}}^{2}(\phi)+\Pi_{Z^{\prime}}^{o o}(0)\right]^{3 / 2}-2 m_{W}^{3}(\phi)-m_{Z}^{3}(\phi)-m_{Z^{\prime}}^{3}(\phi)\right)
\end{aligned}
$$

where $m_{W}^{2}(\phi)=g^{2} \phi^{2} / 4, m_{Z}^{2}=\left(g^{2}+g^{2}\right) \phi^{2} / 4, m_{Z^{\prime}}^{2}=\tilde{g} \phi^{2} / 4$ and $\Pi_{i}^{o o}(0) \sim g_{i}^{2} T^{2}$ such that $g, g^{\prime}$ and $\tilde{g}$ are the $\mathrm{SU}(2), \mathrm{U}(1)$ and $\tilde{U}(1)$ coupling constants, respectively. As explained (4) Notice that, for $m_{Z^{\prime}}>500 \mathrm{GeV}$, the thermal contributions of the extra boson are essentially Boltzmann suppressed and therefore irrelevant for our discussion. 
in ref. [15], these contributions are relevant in the limit $\frac{m_{i}(\phi)}{T} \ll 1$, i.e., $\frac{g_{i} \phi}{2 T} \ll 1$ and tend to vanish when $\frac{m_{i}(\phi)}{T} \simeq 1^{(5)}$. Moreover, in what concerns our discussion, the net effect of the ring diagrams is essentially to reduce the coefficient of $E$, in (1), by a factor $2 / 3[15$, 16].

The survival condition for the baryon asymmetry, namely that B-violating spharelon induced processes drop out of equilibrium after the electroweak phase transition, i.e. $\Gamma_{\beta} \lesssim H$, where

$$
\Gamma_{\not B}=\alpha_{w}^{4} \beta_{o} T^{4} \exp \left[\frac{-E_{s p h}}{T}\right]
$$

( $E_{s p h}$ being the sphaleron energy, $\alpha_{w}$ the weak coupling constant and $\beta_{o} \sim \mathcal{O}(1)$ ), and $\mathrm{H}$ is the expansion rate of the Universe

$$
H^{2}=\frac{8 \pi \rho}{3 M_{P}^{2}}
$$

$\left(M_{P}\right.$ is the Planck mass, $\eta=N_{B}+\frac{7}{8} N_{F}$ and $\rho=\rho(\phi)+\rho(T)$, with $\rho(\phi)=\frac{1}{2} \dot{\phi}^{2}+V(\phi, T)$ and $\left.\rho(T)=\frac{\pi^{2}}{90} \eta T^{4}\right)$, implies that $\frac{\phi}{T}>1$ at the critical field and temperature [8].

The critical field and temperature can be obtained from conditions $\frac{\partial V}{\partial \phi}\left(\phi_{c}, T_{c}\right)=0$ and $V\left(\phi_{c}, T_{c}\right)=V\left(0, T_{c}\right)$ and are given by

$$
\phi_{c}=\frac{4 E T_{c}}{3 \lambda_{T_{c}}}
$$

and

$$
T_{c}=\frac{T_{o}}{\sqrt{1-x}}
$$

where $x=4 E^{2} / 9 D \lambda_{T_{c}}$. We stress once again that, in (10), (11) and in what follows, $\mathrm{E}$ appears always reduced by a factor $2 / 3$ due to the effect of ring diagram contributions. Therefore, the survival condition implies, from eq. (10):

(5) Validity of the perturbation expansion requires $\frac{\alpha_{w} T}{m_{i}(\phi)} \ll 1$ or $\frac{\phi}{T} \gg \frac{g_{i}}{2 \pi} \simeq 0.1$. 


$$
\left(\frac{\phi}{T}\right)_{c}=\frac{4 E}{3 \lambda_{T c}} \gtrsim 1
$$

3. The Higgs mass bounds, obtained from the requiring that $\left(\frac{\phi}{T}\right)_{c} \gtrsim 1$, are shown in Fig. 1 as functions of the top quark mass, in the range $100 \mathrm{GeV}<m_{t}<200 \mathrm{GeV}$, for $m_{Z^{\prime}}=100,200,300,500 \mathrm{GeV}$. We find that one needs $m_{Z^{\prime}}>100 \mathrm{GeV}$ to obtain compatibility with $m_{H} \gtrsim 60 \mathrm{GeV}$ [13]. For $m_{Z^{\prime}}=200 \mathrm{GeV}$, we get $m_{H} \lesssim 100 \mathrm{GeV}$ for all values of $m_{t}$ in the abovementioned range and, for $m_{Z} \gtrsim 300 \mathrm{GeV}$, bounds on $m_{H}$ become fairly insensitive to the top quark mass. For $m_{Z^{\prime}}=500 \mathrm{GeV}$, the Higgs mass bound is weakened up to about $270 \mathrm{GeV}$.

Notice that, allowing $m_{Z^{\prime}} \gtrsim 300 \mathrm{GeV}$ leads to $T_{0}^{2} \leq 0$ and $\lambda_{T} \leq 0$ for certain combinations of $m_{H}$ and $m_{t}$. Negative values of $T_{o}^{2}$ and $\lambda_{T}$ require that expressions (6), (10) and (12) above be rewritten in terms of $\left|T_{o}^{2}\right|$ and $\left|\lambda_{T}\right|$. Singularities encountered for $T_{o}^{2}=0$ and $\lambda_{T}=0$ invalidate, of course, eqs. (6), (10) and (12), but the ensued roots are not relevant for our discussion on the survival of the baryon asymmetry since it turns out, studying the effective potential (1), that near these points the phase transition tends to become second order, thus compromising the whole scenario of baryon asymmetry generation.

Let us now turn to the question of the validity of the thin wall approximation in the class of models we are considering. The relevant parameter is

$$
\epsilon=\frac{T_{c}^{2}-T_{N}^{2}}{T_{c}^{2}-T_{o}^{2}}
$$

where $T_{N}$ is the nucleation temperature. Thin walls satisfy the condition $\epsilon \ll 1$. As discussed in refs. [16], for typical values of SM parameters, the thin wall approximation is not valid; we shall see that this is also the case for the models we are considering. Hence, when discussing the rate of tunnelling from the false to the true vacuum per unit of four-volume [25] 


$$
\frac{\Gamma}{V_{4}}=T^{4}\left(\frac{S_{3}}{2 \pi T}\right)^{3 / 2} \exp \left(-\frac{S_{3}}{T}\right)
$$

one cannot estimate the $O(3)$-symmetric bounce action, $\frac{S_{3}}{T}$, using the thin wall approximation; fortunately, one has the alternative formula of ref. [16]:

$$
\frac{S_{3}}{T}=\frac{87.3 D^{3 / 2}}{E^{2}} \theta^{3 / 2} f\left(\frac{4.5 \lambda_{T_{o}} D}{E^{2}} \theta\right)
$$

where

$$
f(y)=1+\frac{y}{4}\left[1+\frac{2.4}{1-y}+\frac{0.26}{(1-y)^{2}}\right]
$$

and $\theta(T) \equiv \frac{\Delta T}{T}=1-\frac{T_{0}}{T}$. One is interested in the nucleation temperature, $T_{N}$, at which $\Gamma\left(T_{N}\right)=1$. In eq. (14) $V_{4} \sim t^{4} \sim H^{-4}$ and, from eq. (9), with $\rho \approx \rho_{o}=(25 \mathrm{GeV})^{4}$, we get

$$
\Gamma(T)=\frac{9 M_{P}^{4}}{64 \pi^{2} \rho_{o}^{2}} T^{4}\left(\frac{S_{3}}{2 \pi T}\right)^{3 / 2} \exp \left(-\frac{S_{3}}{T}\right) .
$$

The fate of the generated baryon asymmetry will depend on the entropy release, $\Delta$, ensued by the first order phase transition. It is necessary that $\Delta \lesssim 1$, i.e. that $T_{R} \lesssim T_{N}$ since

$$
\Delta \equiv \frac{\eta_{R} T_{R}^{3}}{\eta_{N} T_{N}^{3}}
$$

and $\eta_{R} \gtrsim \eta_{N}, T_{R}$ being the reheating temperature obtained from the condition : $\rho\left(\phi=0, T_{N}\right)=\rho\left(\phi=\sigma, T_{R}\right)$, where $\rho(\phi, T)=V(\phi, T)-T \frac{\partial V(\phi, T)}{\partial T}$.

In the SM, excessive entropy release would represent a serious threat to the baryon asymmetry if the Higgs boson mass were close to the Coleman-Weinberg mass [26]. However, given the present lower bound on $m_{H}$, this problem no longer exists for the SM. In order to check whether this is the case for the models we are considering, we have computed the ratio between the reheating and nucleation temperatures 


$$
\frac{T_{R}}{T_{N}}=\frac{2 D(1-\theta)}{\pi}\left[\frac{15}{\eta_{R}} \frac{(1+\sqrt{1+\gamma}}{\delta}\right]^{1 / 2}
$$

where

$$
\gamma=\frac{\pi^{2}}{30 D^{2}} \eta_{R}\left[\frac{\delta}{(1-\theta)^{2}}+8 B-\lambda_{T N}+\frac{\eta_{N} \pi^{2} \delta^{2}}{120(1-\theta)^{4} D^{2}}\right],
$$

with $\delta=\left(m_{H} / \sigma\right)^{2}-8 B, \eta_{R}=92.5$ and $\eta_{N}=73$, for various combinations of $m_{Z^{\prime}}, m_{H}$ and $m_{t}$; the results are shown in Table 1 , where we also show the corresponding values of $\theta\left(T_{N}\right), \epsilon$ and $S_{3} / T$. We conclude that realistic models with an extra U(1) gauge symmetry are indeed safe in what concerns the possibility of excessive supercooling.

4. We have studied extensions of the SM model with an extra U(1) symmetry in what concerns the preservation of baryon asymmetry after the electroweak phase transition. Demanding the survival of the asymmetry leads to bounds on the Higgs boson mass. While, in the SM, the most recent experimental bound [13] is conflicting with the bound arising from the survival condition (12) for $m_{t} \geq 120 \mathrm{GeV}$ [14], in the extension of the SM we are considering the situation improves considerably provided the mass of the extra $Z^{\prime}$ boson exceeds $200 \mathrm{GeV}$, with some dependence on the top quark mass. For $m_{Z^{\prime}} \gtrsim 300$ $\mathrm{GeV}$, the upper bound on the Higgs boson mass becomes almost insensitive to the top quark mass and for $m_{Z^{\prime}}=500 \mathrm{GeV}$, it can be raised up to $270 \mathrm{GeV}$. 


\section{References}

[1] A.D. Sakharov, JEPT Lett. 5(1967)24.

[2] M Yoshimura, Phys. Rev. Lett. 41(1978)281; Phys. Lett B88(1979)294;

A. Yu Ignatiev, N.V. Krasnikov, V.A. Kuzmin and A.N. Tavkhelidze, Phys. Lett. B76(1978)436;

D. Toussaint, S. B. Treiman, F. Wilczek and A. Zee, Phys. Rev. D19(1979)1036;

S. Weinberg, Phys. Rev. Lett. 42(1979)850.

[3] A.D. Linde, Phys. Lett. B70(1977)306;

S. Dimopoulos and L. Susskind, Phys. Rev. D18(1978)4500.

[4] M. Claudson, L.J. Hall and I. Hinchliffe, Nucl. Phys. B241(1984)309;

G. Lazarides, C. Panagiatakopoulos and Q. Shaf, Phys. Rev. Lett. 56(1986)557;

K. Yamamoto, Phys. Lett. B168(1986)341;

O. Bertolami and G.G. Ross, Phys. Lett. B183(1987)163;

A. Cline and S. Raby, Phys. Rev. D43(1991)1781;

S. Mollerach and E. Roulet, Phys. Lett. B281(1992)303.

[5] I. Affleck and M. Dine, Nucl. Phys. B249(1985)361.

[6] A.D. Dolgov and Ya. B. Zel'dovich, Rev. Mod. Phys. 53(1981)1;

A. Cohen and D. Kaplan, Phys. Lett. B199(1987)251.

[7] N.S. Manton, Phys. Rev. D28(1983)2019;

F.R. Klinkhamer and N.S. Manton, Phys. Rev. D30(1984)2212.

[8] V.A. Kuzmin, V.A. Rubakov and M.E. Shaposhnikov, Phys. Lett. B155(1985)36.

[9] M.E. Shaposhnikov, JEPT Lett. 44(1986)465, Nucl. Phys. B287(1987)757, B299 (1988)797;

M. Fukugita and T. Yanagida, Phys. Lett. B174(1986)45;

L. McLerran, Phys. Rev. Lett. 62(1989)1075;

N. Turok and J. Zadrozny, Phys. Rev. Lett. 65(1990)2331; Nucl. Phys. B358(1991) 471; 
M. Dine, P. Huet, R. Singleton and L. Susskind, Phys. Lett. B257(1991)351;

J. McDonald, Lisbon Preprint IFM-6/93.

[10] A. Cohen, D. Kaplan and A. Nelson, Nucl. Phys. B349(1991)727; Phys. Lett. B263(1991)86.

[11] G.R. Farrar and M.E. Shaposhnikov, Phys. Rev. Lett. 70(1993)2833; Preprint CERNTH. 6734/93/RU-93-11,1993.

[12] M.E. Shaposhnikov, Phys.Lett B277(1992)324;B282(1992)483(E).

[13] Aleph Collaboration, Preprint CERN-PPE/93-110.

[14] K.E.C. Benson, Phys. Rev. D48(1993)2456.

[15] M.E. Carrington, Phys. Rev. D45(1992)2933.

[16] M. Dine, R.G. Leigh, P. Huet, A.D. Linde and D. Linde, Phys. Rev. D46(1992)550; Phys. Lett. B283(1992)319.

[17] M. Dine, P. Huet and R. Singleton Jr., Nucl. Phys. B375(1992)625.

[18] A.G. Cohen and A.E. Nelson, Phys. Lett. 297B(1992)111.

[19] G. W. Anderson and L.J. Hall, Phys. Rev. D45(1992)2685.

[20] L.S. Durkin and P. Langacker, Phys. Lett. B166(1986)436.

[21] G. Altarelli et al., Preprint CERN-TH 6947/93, UGVA-DTP 1993/07-827.

[22] L3 Collaboration, Preprint CERN PPE/93-44.

[23] F. Abe et al. (CDF Collaboration), Phys. Rev. Lett. 68(1992)1463.

[24] M. Sher, Phys. Rep. 179(1989)273.

[25] A.D. Linde, Phys. Lett. B100(1981) 132; Nucl. Phys. B216(1983)421.

[26] E. Witten, Nucl. Phys. B177(1981)477. 
Figure and Table Captions

Fig.1 : Comparison of Higgs mass bounds versus the top mass, for the Standard Model (dashed curve) and models with a $Z^{\prime}$, for $m_{Z^{\prime}}=100,200,300,500 \mathrm{GeV}$ (full curves).

Table 1 : Values of relevant quantities in the Standard Model (first line) and in models with a $Z^{\prime}$. 


\begin{tabular}{ccccccc}
$m_{Z^{\prime}}(\mathrm{GeV})$ & $m_{H}(\mathrm{GeV})$ & $m_{t}(\mathrm{GeV})$ & $\theta\left(T_{N}\right)$ & $\epsilon$ & $S_{3} / T$ & $T_{R} / T_{N}$ \\
\hline$S M$ & 30 & 120 & 0.0133 & 0.247 & 193.9 & 0.021 \\
100 & 40 & 140 & 0.0135 & 0.188 & 193.9 & 0.038 \\
200 & 70 & 180 & 0.0287 & 0.142 & 192.3 & 0.102 \\
300 & 125 & 190 & 0.0612 & 0.133 & 190.6 & 0.221 \\
500 & 250 & 170 & 0.1936 & 0.249 & 187.5 & 0.415 \\
& & & & & & \\
\hline
\end{tabular}

Table 1

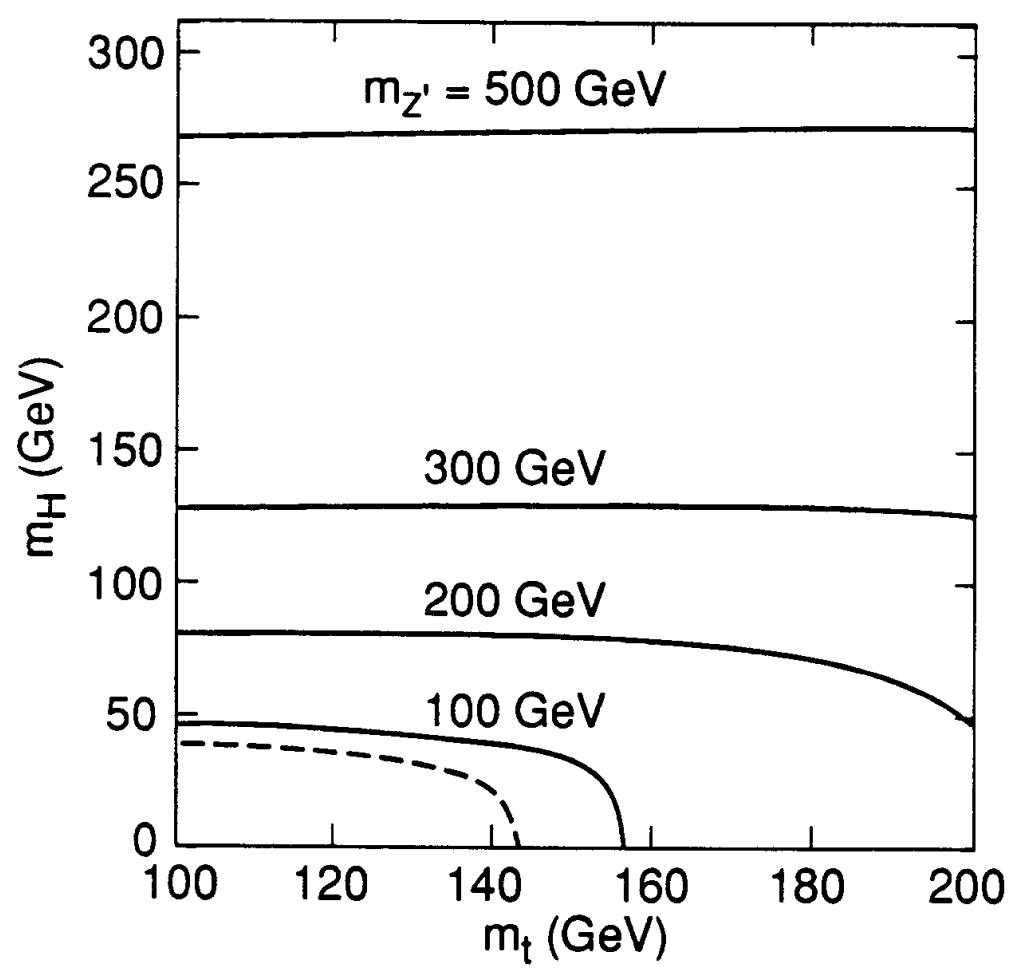

Fig. 1 